\title{
Factores asociados al desarrollo de infección urinaria de origen comunitario causada por Escherichia coli resistente a fluoroquinolonas
}

\author{
Verónica Seija, Victoria Frantchez, Verónica Ventura, Marcos Pintos y Mariana González
}

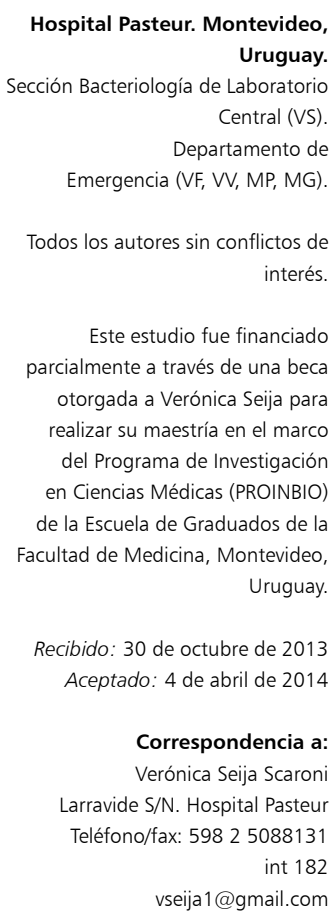

Hospital Pasteur. Montevideo, Uruguay. Sección Bacteriología de Laboratorio Central (VS) Departamento de Emergencia (VF, VV, MP, MG)

Todos los autores sin conflictos de interés.

Este estudio fue financiado parcialmente a través de una beca otorgada a Verónica Seija para realizar su maestría en el marco

del Programa de Investigación en Ciencias Médicas (PROINBIO) de la Escuela de Graduados de la Facultad de Medicina, Montevideo,

Uruguay.

Recibido: 30 de octubre de 2013 Aceptado: 4 de abril de 2014

Correspondencia a: Verónica Seija Scaron Larravide S/N. Hospital Pasteur Teléfono/fax: 59825088131

vseija1@gmail.com

\section{Introducción}

L a infección del tracto urinario (ITU) constituye una de las consultas más frecuentes en el primer nivel de atención de salud ${ }^{1}$. Escherichia coli es el microorganismo uropatógeno más frecuente, causante de $75-90 \%$ de las ITU ${ }^{1}$. La terapia para los episodios de ITU aguda es inicialmente empírica y en el caso de ITU no complicada, a menudo no se indican estudios para establecer la etiología. Los antimicrobianos recomendados por Infectious Diseases Society of America (IDSA) para el tratamiento de la cistitis no complicada en adultos incluyen nitrofurantoína, cotrimoxazol y fosfomicina trometamol ${ }^{2}$. Para los episodios de pielonefritis no complicada, IDSA recomienda el uso de ciprofloxacina siempre que los niveles locales de resistencia no superen el $10 \%{ }^{2}$.

En Uruguay lo niveles de resistencia a cotrimoxazol superan el $30 \%$ desde hace muchos años ${ }^{3,4}$, lo cual es una de las razones que ha provocado que las fluoroquinolonas (FQ) sean la terapéutica empírica más frecuentemente utilizada para el tratamiento de los episodios de ITU complicada y no complicada en adultos ${ }^{5,6}$. En los últimos años se ha observado, en nuestro país, como en el resto de Latinoamérica, un número creciente de cepas de $E$. coli resistentes a $\mathrm{FQ}^{7,8}$.

La instauración de un adecuado tratamiento antimicrobiano empírico es una de las medidas de mayor importancia para disminuir la morbi-mortalidad en este tipo de infecciones ${ }^{9}$. El objetivo del presente trabajo fue conocer los factores de riesgo asociados al desarrollo de una ITU de origen comunitario causada por E. coli resistente a fluoroquinolonas (ECRFQ) de manera de contribuir a mejorar la selección del tratamiento antimicrobiano empírico más adecuado.

\section{Material y Método}

\section{Diseño epidemiológico}

Estudio descriptivo, observacional, transversal, donde los pacientes incluidos fueron captados en forma prospectiva. Para determinar los factores de riesgo se realizó un estudio de casos/control donde los casos estuvieron constituidos por los pacientes que desarrollaron ITU causada por ECRFQ y aquellos sin esta condición se consideraron controles.

\section{Población}

Pacientes mayores de 15 años que consultaron por ITU adquirida en la comunidad, en el Departamento de Emergencia (DE) del Hospital Pasteur, perteneciente a la Administración de Servicios de Salud del Estado (Montevideo-Uruguay), entre junio de 2007 y septiembre de 2011. 
Para ser consideradas sólo las ITU adquiridas en la comunidad, se excluyeron los pacientes con antecedentes de internación en el mes previo a la consulta. También se excluyeron pacientes portadores de algún tipo de instrumentación sobre la vía urinaria y pacientes institucionalizados.

\section{Definiciones}

Se definió infección del tracto urinario como: recuento bacteriano (monomicrobiano) en orina igual o mayor de $10.000 \mathrm{ufc} / \mathrm{ml}$, con al menos un síntoma de ITU (disuria, aumento de frecuencia o urgencia miccional $)^{10}$.

Se incluyeron tanto los episodios del tracto urinario bajo (cistitis) como del tracto urinario alto (pielonefritis). Para cistitis se utilizó la definición de ITU antes mencionada y pielonefritis se definió como aquella ITU que se presentó con fiebre y/o dolor lumbar.

Se consideró ITU complicada a aquella que se presentó en pacientes con al menos una de las siguientes condiciones: sexo masculino, más de tres episodios de ITU en el último año (ITU recurrente), embarazo, monorreno/a, diabetes mellitus, insuficiencia renal crónica, antecedentes de trasplante de órgano sólido, inmunocompromiso y uropatía obstructiva donde se incluyeron todas las alteraciones del tracto urinario que cursaban con obstrucción del mismo (por ejemplo: litiasis urinaria o patología prostática). Estas condiciones fueron investigadas en el interrogatorio como antecedentes del paciente. No se realizaron estudios adicionales que podrían haber detectado nuevas situaciones de complicación al momento de la consulta.

\section{Estudio microbiológico}

La recolección de la muestra de urocultivo se realizó en el DE, la indicación del mismo quedó a criterio del médico tratante quien completó la solicitud de urocultivo con bacteriuria cuantitativa que incluía: datos patronímicos, criterios para ITU complicada, procedimiento de recolección de la muestra, antecedentes de internación en el último año (excluyendo los internados en el mes previo a la consulta) y uso de antimicrobianos en los últimos tres meses. A todos los pacientes se les explicó el procedimiento para la toma de muestra (chorro medio). Las muestras fueron transportadas al Laboratorio Central del Hospital Pasteur antes de las dos horas de emitidas y, en caso de ser necesario, fueron conservadas a $4^{\circ} \mathrm{C}$ por hasta $24 \mathrm{~h}$ antes de su procesamiento. Las muestras se sembraron en agar cistina-lactosa deficiente en electrolitos con asa calibrada. Se realizó una lectura a las 18-24 h de incubación. Aquellas muestras que mostraron escaso desarrollo o que provenían de pacientes inmunocomprometidos fueron incubadas hasta $48 \mathrm{~h}$. Aquellas muestras que mostraron un desarrollo significativo (mayor o igual a $10.000 \mathrm{ufc}$ / $\mathrm{ml}$ ) de un cultivo puro fueron estudiadas ${ }^{10}$ mediante la identificación del agente y estudio de sensibilidad por disco difusión de acuerdo a normativa del "Clinical and Laboratory Standards Institute" (CLSI) ${ }^{11}$. En el caso de $E$. coli se registraron los datos de susceptibilidad a los siguientes antimicrobianos: ampicilina, ampicilina/ sulbactam, cefuroxima, ceftriaxona, ceftazidima, ácido nalidíxico, ciprofloxacina, gentamicina, amikacina, nitrofurantoína y cotrimoxazol. La detección de $\beta$-lactamasas de espectro extendido (BLEE) se realizó mediante la colocación de los discos de cefalosporinas de tercera generación a $25 \mathrm{~mm}$ del disco de amoxicilina/ác. clavulánico y se buscó el ensachamiento del halo de sensibilidad a las cefalosporinas, como indicativo de presencia de $\mathrm{BLEE}^{12}$.

\section{Análisis estadístico}

Se realizó con el paquete estadístico SPSS para Windows. La comparación de variables continuas se realizó mediante el test de Student. La relación entre dos variables categóricas se estudió aplicando la prueba de $\chi^{2}$ y el test exacto de Fisher según correspondiera. Se realizó análisis multivariado para determinar los factores predictores de ITU ECRFQ. El valor de significación fue 0,05.

\section{Ética}

El protocolo de investigación fue aprobado por el Comité de Ética del Hospital Pasteur. El manejo de los datos obtenidos se realizó manteniendo el anonimato de los individuos.

\section{Resultados}

En el período analizado se diagnosticaron 525 pacientes con ITU de origen comunitario. La media de edad fue $43,5 \pm 20,1$ años, $442(84,2 \%)$ de sexo femenino, 320 $(61 \%)$ se presentaron con ITU alta y $254(48,4 \%)$ con ITU complicada. Para los pacientes con ITU no complicada (n: 271) la media de edad fue $35,4 \pm 17,8$ años y $51,9 \pm$ 19,1 para aquellos con IU complicada $(\mathrm{p}<0,001)$.

En 434 pacientes $(82,7 \%)$ el microorganismo encontrado fue E. coli. En Tabla 1 se puede ver la tasa de resistencia de estas cepas. Los aislados de E. coli resistentes a FQ fueron significativamente más resistentes a cefuroxima $(11,8 \%$ versus $0,5 \%, p<0,001)$, ceftriaxona $(8,5 \%$ versus $0,3 \%, p<0,001)$ y la detección fenotípica de BLEE fue también significativamente más frecuente $(8,5 \%$ versus $0,3 \%, \mathrm{p}<0,001)$.

En el subgrupo de pacientes con pielonefritis por $E$. coli (n: 272) la resistencia a FQ fue $12,1 \%$ no habiendo diferencias significativas con el nivel de resistencia (16,0\%) encontrado en los 162 pacientes con cistitis aguda (p: 0,25). En aquellos pacientes con pielonefritis no complicada (n: 140) la resistencia a FQ fue 5,0\% mientras en aquellos con pielonefritis complicada (n: 132) 19,7\% 


\begin{tabular}{|c|c|c|}
\hline Antimicrobiano & $\begin{array}{c}\text { Frecuencia } \\
\text { absoluta }\end{array}$ & $\begin{array}{c}\text { Frecuencia } \\
\text { relativa \% }\end{array}$ \\
\hline Ampicilina & 229 & 52,7 \\
\hline Ampicilina/sulbactam & 93 & 21,4 \\
\hline Cefuroxima & 9 & 2,1 \\
\hline Ceftriaxona & 6 & 1,4 \\
\hline Ceftazidima & 2 & 0,5 \\
\hline Nitrofurantoina & 9 & 2,1 \\
\hline Cotrimoxazol & 146 & 33,6 \\
\hline Ac nalidíxico & 87 & 20,0 \\
\hline Ciprofloxacina & 59 & 13,6 \\
\hline Gentamicina & 17 & 3,9 \\
\hline Amikacina & 1 & 0,2 \\
\hline Carbapenems & 0 & 0 \\
\hline Detección fenotípica de BLEE & 6 & 1,4 \\
\hline
\end{tabular}

$(\mathrm{p}<0,001)$. Asimismo, en quienes tuvieron cistitis aguda no complicada (n: 89 ) fue 7,9\% y en aquellos con cistitis complicada (n: 73 ) 26,0\% (p: 0,004).

En el período 2010-2011 se detectaron por primera vez seis cepas de E. coli que, fenotípicamente, eran productoras de BLEE.

En la Tabla 2 se observan las características de los pacientes según la presencia o no de ITU por ECRFQ y los resultados del análisis multivariado que identificó como factores de riesgo independientes para el desarrollo de ITU por ECRFQ que el paciente fuera mayor de 60 años, portador de uropatía obstructiva, con antecedentes de ITU recurrente y/o con antecedentes de uso de FQ en los tres meses previos a la consulta.

\section{Discusión}

En nuestra serie, al igual que en otras, los episodios de ITU fueron más frecuentes en el sexo femenino ${ }^{13-15}$. La resistencia global de E. coli a fluoroquinolonas en el presente trabajo fue $13,6 \%$, menor para los aislados de ITU no complicada y mayor para los de ITU complicada. No encontramos diferencias estadísticamente significativas

Tabla 2. Factores de riesgo para ITU en adultos por Escherichia coli resistente a FQ: análisis univariado y multivariado. Hospital Pasteur, Montevideo, Uruguay

\begin{tabular}{|c|c|c|c|c|c|c|}
\hline \multirow[b]{2}{*}{ Variable } & \multirow[b]{2}{*}{$\begin{array}{l}\text { E. coli } \mathrm{FQ} \text { resistente } \\
\mathrm{n}=59(\%)\end{array}$} & \multicolumn{2}{|c|}{ Análisis univariado } & \multirow[b]{2}{*}{ Valor $p$} & \multicolumn{2}{|c|}{ Análisis multivariado } \\
\hline & & $\begin{array}{c}\text { E. coli FQ sensible } \\
n=375(\%)\end{array}$ & $\begin{array}{c}\text { OR } \\
\text { (IC 95\%) }\end{array}$ & & $\begin{array}{l}\text { OR ajustado } \\
\text { (IC 95\%) }\end{array}$ & Valor $p^{d}$ \\
\hline Sexo masculino & $11(18,6)$ & $66(17,6)$ & $1,33(0,65-2,73)$ & $0,429^{a}$ & & \\
\hline Edad media (años) $\pm \mathrm{DE}$ & $52,4 \pm 20,0$ & $42,4 \pm 19,5$ & - & $<0,001^{b}$ & & \\
\hline Edad mayor a 60 años & $23(39,0)$ & $73(19,5)$ & $2,64(1,47-4,73)$ & $0,001^{\mathrm{a}}$ & $2,52(1,35-4,72)$ & 0,004 \\
\hline Pielonefritis aguda & $33(55,9)$ & $239(63,7)$ & $0,72(0,41-1,26)$ & $0,25^{a}$ & & \\
\hline ITU complicada & $45(76,3)$ & $160(42,7)$ & $4,32(2,29-8,14)$ & $<0,001^{a}$ & & \\
\hline Diabetes mellitus & $13(22,0)$ & $40(10,7)$ & $2,41(1,20-4,85)$ & $0,011^{\mathrm{a}}$ & & \\
\hline ITU recurrente & $22(37,3)$ & $53(14,1)$ & $3,60(1,97-6,58)$ & $<0,001^{a}$ & $2,98(1,55-5,76)$ & 0,001 \\
\hline Uropatía obstructiva & $16(27,1)$ & $45(12,0)$ & $2,79(1,45-5,36)$ & $0,002^{\mathrm{a}}$ & $2,09(1,03-4,28)$ & 0,042 \\
\hline Insuficiencia renal crónica & $4(6,8)$ & $6(1,6)$ & $4,54(1,24-16,62)$ & $0,013^{c}$ & & \\
\hline Inmunocompromiso & $1(1,7)$ & $10 \quad(2,7)$ & $0,64(0,08-5,08)$ & 0,669 & & \\
\hline Antecedentes de uso de AM en últimos tres meses & $24(40,7)$ & $58(15,5)$ & $3,74(2,07-6,74)$ & $<0,001^{\text {a }}$ & & \\
\hline Antecedentes de uso de FQ en últimos tres meses & $15(25,4)$ & $17(4,5)$ & $7,29(3,40-15,62)$ & $<0,001^{\text {a }}$ & $4,27(1,88-9,71)$ & 0,001 \\
\hline Antecedentes de uso de $\beta$-lactámicos en últimos tres meses & $3(5,1)$ & $16(4,3)$ & $1,20(0,34-4,26)$ & $0,73^{c}$ & & \\
\hline Antecedentes de uso de cotrimoxazol en últimos tres meses & $1(1,7)$ & $6(1,6)$ & $1,06(0,13-8,97)$ & $1^{\mathrm{c}}$ & & \\
\hline Antecedentes de internación en el último año & $5(16,3)$ & $13(3,4)$ & $2,27(0,73-7,06)$ & $0,15^{\mathrm{a}}$ & & \\
\hline
\end{tabular}


entre el porcentaje de cepas resistentes a FQ provenientes de aislados causantes de pielonefritis y de cistitis aguda, como sí lo han hecho otros autores ${ }^{16}$.

Nuestra cifra de resistencia global a FQ en E.coli de origen urinario $(13,6 \%)$, es menor a la reportada por otros países de Latinoamérica ${ }^{7}$.

En relación a las cifras reportadas por E.U.A., la resistencia global a FQ en aislados de $E$. coli urinarios ha tenido un incremento significativo entre los años 2000, cuando apenas era 3\% y 2010 cuando alcanzó $17,1 \%{ }^{17}$.

Si tenemos en cuenta sólo las cifras de resistencia a FQ en ITU no complicada, nuestra tasa de resistencia es discretamente menor a la reportada en España, Rusia, Italia y Brasil ${ }^{18}$ y cercana a las tasas de resistencia reportadas por Portugal ${ }^{19}$.

Sin embargo, la comparación de tasas de resistencia a FQ en aislados urinarios de E. coli es dificultosa debido a los diferentes criterios de inclusión y exclusión que tienen los estudios a lo cual se suma que muchos se basan en datos de laboratorio sin tener en cuenta variables clínicas ${ }^{20}$.

En nuestro estudio, la detección fenotípica de BLEE así como la resistencia a ceftriaxona fue significativamente más frecuente en aislados de ECRFQ, no alcanzando cifras que impidan el uso de este antimicrobiano como terapia empírica en episodios ITU de origen comunitario.

Los principales mecanismos de resistencia a FQ en E. coli son las mutaciones puntuales en los genes gyr $A$ y $\operatorname{par} C$, lo cual provoca alteraciones del sitio blanco de las $\mathrm{FQ}^{21}$. Recientemente se han descrito tres nuevos genes que codifican mecanismos de resistencia a quinolonas que, a diferencia del mecanismo anterior, son transferibles horizontalmente: $q e p A^{22}$, gen $q n r$ y gen $a a c\left(6^{\prime}\right)-I b c r^{21}$. Estos dos últimos mecanismos ya han sido descritos, en nuestro país, en cepas de origen nosocomial ${ }^{23,24}$.

Conocer las características de los pacientes con ITU por ECRFQ es importante dado que la falla clínica en el tratamiento de la ITU ha sido asociada a resistencia in vitro ${ }^{9}$. En nuestro análisis, luego de ajustar los factores de riesgo del análisis univariado, el desarrollo de ITU causada por ECRFQ estuvo asociado a edad mayor de 60 años, presencia de uropatía obstructiva, ITU recurrente y antecedentes de uso de FQ en los tres meses previos a la consulta.

A pesar que varios trabajos analizan los factores de riesgo asociados al desarrollo de ITU por bacilos gramnegativos resistente a $\mathrm{FQ}^{13,25-30}$ pocos analizan, exclusivamente, infecciones de origen comunitario ${ }^{14,15,29}$ y ninguno analizó, como lo hizo nuestro estudio, pacientes con ITU de origen comunitario identificados en forma prospectiva como tampoco excluyeron aquellos que pudieran tener factores de confusión tales como portación de catéter vesical y/o institucionalizados.

En relación a la mayor edad como factor de riesgo, algunos trabajos ${ }^{13,15,29}$ la encuentran en el análisis uni- variado, no así en el multivariado pero Arslan y cols. ${ }^{14}$, encontraron que tener una edad mayor a 50 años era un factor de riesgo independiente. En el mismo sentido nuestro trabajo encontró lo mismo pero utilizó el límite de 60 años.

La presencia de ITU complicada ha sido encontrada como factor de riesgo en trabajos previos ${ }^{14} \mathrm{y}$ también la ITU recurrente ${ }^{15,29}$. Ena y cols. ${ }^{30}$, encontraron que la presencia de anormalidades del tracto urinario era un factor de riesgo para el desarrollo de ITU por ECRFQ. En nuestro trabajo utilizamos el termino uropatía obstructiva para hablar de pacientes con anormalidades del aparato urinario que cursaban con obstrucción del mismo.

La mayoría de los trabajos han encontrado que la exposición previa a FQ es un factor significativo para el desarrollo de ITU causada por ECRFQ ${ }^{14,15,29}$, de la misma manera que lo ha hecho nuestro análisis. En nuestro caso, el haber recibido otros antimicrobianos distintos a FQ no fue un factor de riesgo independiente para el desarrollo de ITU por ECRFQ como sí lo ha sido en otros estudios ${ }^{25-27}$. Esto podría ser debido a que estos trabajos incluyeron pacientes hospitalizados y quizás en estas poblaciones se utilicen mayor número de antimicrobianos que pueden seleccionar mecanismos de resistencia a FQ.

La asociación entre emergencia de resistencia a FQ y consumo de FQ ha sido reportado en numerosos trabajos $^{14,31}$. Datos de 2007 muestran que nuestro país tuvo un consumo de quinolonas que no llegó a $1 \mathrm{DDD} / 1.000$ habitantes/día ${ }^{32}$, muy lejos de las 2,5 DDD/1.000 habitantes/día que se vieron en España durante $2005^{33}$. Sin embargo, nuestro consumo de quinolonas de 2007 fue el doble que en 1997, lo que podría explicar, en parte, la tasa de resistencia a estos agentes encontrada en el presente trabajo. En ese sentido, Ena y cols. ${ }^{34}$ observaron, concomitantemente con la triplicación en el consumo de FQ, un aumento en la tasa de resistencia a FQ en E. coli, de 3 a $20 \%$.

Otros trabajos demuestran que no siempre se puede establecer una clara correlación entre consumo y resistencia a quinolonas en $E$. coli ${ }^{35}$. En ese sentido, se ha demostrado la diseminación de grupos clonales de cepas de $E$. coli resistentes a $\mathrm{FQ}^{36,37}$, los cuales podrían tener un reservorio en animales y/o alimentos ${ }^{38}$.

Nuestro estudio tiene algunas limitaciones. En primera instancia no incluimos episodios de ITU con recuentos menores a $10.000 \mathrm{ufc} / \mathrm{ml}$. En segundo lugar, correlacionamos los hallazgos clínicos con los hallazgos microbiológicos pero no con los resultados del examen de orina (presencia de leucocitos). En tercer lugar, al momento de la consulta, no realizamos exámenes complementarios adicionales que podrían haber detectado más factores de complicación. En cuarto lugar, no hicimos una diferenciación entre pacientes con uropatía obstructiva por litiasis o patología prostática así como dentro de estos 
últimos aquellos con antecedentes de instrumentación. Esto, quizás, nos hubiera permitido realizar una mejor categorización de los factores de riesgo.

En suma:

De acuerdo a nuestros resultados, el uso empírico de FQ aún es adecuado para el tratamiento de los episodios de ITU no complicadas, dados los bajos niveles de resistencia detectados. De todas maneras, creemos que las mismas deben reservarse para los episodios de pielonefritis aguda no complicada donde existen pocas opciones terapéuticas de uso oral, no así en cistitis aguda donde hay opciones disponibles como nitrofurantoína y fosfomicina. Sin embargo, en pacientes con ITU recurrente, uropatía obstructiva, mayores de 60 años o uso previo de FQ, se deben buscar alternativas terapéuticas a las FQ ya que existe riesgo elevado de encontrar a ECRFQ como causa del episodio de ITU.

Es necesario continuar, por un lado, con el monitoreo de los patrones de resistencia locales y regionales, especialmente a través de estudios clínicos y epidemiológicos y por otro, con el uso prudente de las quinolonas, de manera de intentar frenar el aumento de la resistencia a estos agentes.

Agradecimientos. A todos los médicos, residentes e internos del Departamento de Emergencia del Hospital Pasteur por el tiempo que se tomaron en llenar el protocolo que sirvió de base para este estudio y a Cristina Bazet y Rafael Vignoli por la revisión del proyecto de investigación.

\section{Resumen}

En Uruguay, las fluoroquinolonas (FQ) son el tratamiento empírico más utilizado para episodios de infección del tracto urinario (ITU), reportándose tasas crecientes de resistencia a las mismas. El objetivo fue conocer los factores de riesgo asociados al desarrollo de una ITU de origen comunitario causada por Escherichia coli resistente a fluoroquinolonas (ECRFQ). Se llevó adelante un estudio descriptivo, transversal de una población de 525 pacientes con ITU de origen comunitario, identificados en forma prospectiva, que consultaron en el Dpto. de Emergencia del Hospital Pasteur. En 434 pacientes $(82,7 \%)$ la causa de la ITU fue E. coli. Se realizó análisis multivariado que identificó como factores de riesgo independientes para el desarrollo de ITU por ECRFQ que el paciente fuera mayor de 60 años (OR 2,52 IC 95\% 1,35-4,72), portador de uropatía obstructiva (OR 2,09 IC 95\% 1,034,28 ) con antecedentes ITU recurrente (OR 2,98 IC 95\% 1,55-5,76) y/o de uso de FQ en los tres meses previos (OR 4,27 IC 95\% 1,88-9,71). En aquellos pacientes con alguno de estos factores de riesgo existe riesgo elevado de encontrar a ECRFQ como causa del episodio de ITU y por tanto se deben buscar alternativas terapéuticas diferentes de las FQ.

\section{Referencias bibliográficas}

1.- Litza J A, Brill J R. Urinary tract infections. Prim Care Clin Office Pract 2010; 37: 491-507.

2.- Gupta K, Hooton T M, Naber K G, Wullt B, Colgan R, Miller L G, et al. International Clinical Practice Guidelines for the Treatment of Acute Uncomplicated Cystitis and Pyelonephritis in Women: A 2010 Update by the Infectious Diseases Society of America and the European Society for Microbiology and Infectious Diseases. Clin Infect Dis 2011; 52 (5): e103-20.

3.- Organización Panamericana de la Salud. Informe Anual de la Red de Monitoreo/ Vigilancia de la Resistencia Antibiótica. Brasilia. 2005.

4.- Pedreira W, Anzalone L, Alvez M, Cafferatta A. Fosfomicina trometamol. Una opción terapéutica válida en infecciones urinarias bajas. Rev Med Uruguay 2003; 19: 107-16.

5.- Facal J. Infección urinaria. Carta infectológica 2006; 6: 7-12.

6.- Ventura V, Seija V, González M, Ventura S, López M, Frantchez V. Prescripción de antibióticos en episodios de infección urinaria comunitaria del adulto. En XV Congreso Panamericano de Infectología, Punta del Este, Uruguay, 7-11 abril, 2011.

7.- $\quad$ Andrade S S, Sader H S, Jones R N, Pereira A S, Pignatari A, Gales A C. Increased resistance to first-line agents among bacterial pathogens isolated from urinary tract infections in Latin America: time for local guidelines? Mem Inst Oswaldo Cruz 2006; 101: 741-8.

8.- Seija V, Frantchez V, Pintos M, Bataglino M N, Torales M, Díaz A, et al. Etiología de la infección urinaria de adquisición comunitaria y perfil de susceptibilidad de Escherichia coli a los principales agentes antimicrobianos. Rev Med Urug 2010; 26: 14-24.

9.- Gagliotti C, Buttazzi R, Sforza S, Moro M L, Romagna E. Antibiotic Resistance Study Group. Resistance to fluoroquinolones and treatment failure/short-term relapse of communityacquired urinary tract infections caused by Escherichia coli. J Infect 2008; 57 (3): 179-84.

10.- Domingo A D, Cacho J, Coira Nieto A, Lepe Jiménez J A. Diagnóstico microbiológico de las infecciones del tracto urinario. Recomendaciones de la Sociedad Española de Enfermedades Infecciosas y Microbiología Clínica. http://www.seimc.org/documentos/ protocolos/microbiologia/. (Accedido el 1 de marzo de 2013).

11.- Clinical Laboratory Standard Institute. Performance Standards for Antimicrobial Susceptibility Testing; Seventeenth Informational Supplement 2007. CLSI document M100-S17.

12.- Galas M, Red WHONET Argentina. Grupo KES. Servicio de Antimicrobianos. Instituto Nacional de Enfermedades Infecciosas ANLIS "Dr. Carlos G. Malbrán”. http://antimicrobianos. com.ar/2013/02/boletin-13-grupo-kes/ (accedido el 20 de marzo de 2014).

13.- Vásquez G A, Siu H R, Luna E M, Reyes K, Zervos M J. Risk Factors for quinolone-resistant Escherichia coli urinary tract infection. Infect Dis Clin Pract 2009; 17: 309-13.

14.- Arslan H, Azap O K, Ergonul O, Timurkaynak F. Risk factors for ciprofloxacin resistance among Escherichia coli strains isolated from community-acquired urinary tract infections in Turkey. J Antimicrob Chemother 2005; 56 : 914-8.

15.- Colodner R, Kometiani I, Chazan B, Raz R. Risk factors for community-acquired urinary tract infection due to quinolone-resistant $E$. coli. Infection 2008; 36: 41-5. 
16.- Velasco M, Horcajada J P, Mensa J, Moreno-Martínez A, Vila J, Martínez J A, et al. Decreased invasive capacity of quinolone resistant Escherichia coli in urinary tract infection. Clin Infect Dis 2001; 33: 1682-6.

17.- Sánchez G V, Master R N, Karlowsky J, Bordon J M. In vitro antimicrobial resistance of urinary Escherichia coli isolates among U.S. outpatients from 2000 to 2010. Antimicrob Agents Chemother 2012; 56: 2181-3.

18.- Schito G C, Naber K G, Botto H, Palou J, Mazzei T, Gualco L, et al. The ARESC study: an international survey on the antimicrobial resistance of pathogens involved in uncomplicated urinary tract infections. Int $\mathrm{J}$ Antimicrob Agents 2009; 34: 407-13.

19.- Kahlmeter G, Poulsen H O. Antimicrobial susceptibility of Escherichia coli from community-acquired urinary tract infections in Europe: the ECO-SENS study revisited. Int J Antimicrob Agents 2012; 39: 45-51.

20.- Lopardo G, Fridman D, González Arzac M, Calmaggi A, Smayevsky J, Podesta O, et al. Uropathogen resistance: are laboratorygenerated data reliable enough? J Chemother 2007; 19: 33-7.

21.- Robicsek A, Jacoby G A, Hooper D C. The worldwide emergence of plasmid-mediated quinolone resistance. Lancet Infect Dis 2006; 6: 629-40.

22.- Yamane K, Wachino J, Suzuki S, Kimura K, Shibata N, Kato N, et al. New plasmid-mediated fluoroquinolone efflux pump, QepA, found in an Escherichia coli clinical isolate, Antimicrob. Agents Chemother 2007; 51: 3354-60.

23.- Cordeiro N, Robino L, Medina J, Seija V, Bado I, García V, et al. Ciprofloxacin-resistant enterobacteria harboring the aac(6')-Ib-cr variant isolated from feces of inpatients in an intensive care unit in Uruguay. Antimicrob Agents Chemother 2008; 52: 806-7.

24.- Bado I, Robino L, García V, Cordeiro N, Seija V, Gutkind G, et al. First detection of qnr genes in Uruguay. In: 48th Annual ICAAC/ IDSA 46th Annual Meeting, Washington DC, 2008.

25.- Levin P D, Fowler R A, Guest C, Sibbald W J, Kiss A, Simor A E. Risk factors associated with resistance to ciprofloxacin in clinical bacterial isolates from intensive care unit patients. Infect Control Hosp Epidemiol 2007; 28: 331-6.

26.- Rattanaumpawan P, Tolomeo P, Bilker W B, Fishman N O, Lautenbach E. Risk factors for fluoroquinolone resistance in Gram-negative bacilli causing healthcare-acquired urinary tract infections. J Hosp Infect 2010; 76: 324-7.

27.- Nicolettia J, Kustera S P, Sulserb T, Zbindenc $\mathrm{R}$, Ruefa C, Ledergerbera B, et al. Risk factors for urinary tract infections due to ciprofloxacinresistant Escherichia coli in a tertiary care urology department in Switzerland. Swiss Med Wkly 2010; 140: w13059.

28.- Blaettler L, Mertz D, Frei R, Elzi L, Widmer F, Battegay M, et al. Secular trend and risk factors for antimicrobial resistance in Escherichia coli isolates in Switzerland 1997-2007. Infection 2009; 37: 534-9.

29.- Killgore K, March K, Guglielmo J. Risk factors for community-acquired ciprofloxacin-resistant Escherichia coli urinary tract infection. Ann Pharmacother 2004; 38: 1148-52.

30.- Ena J, Amador C, Martínez C, Ortiz de la Tabla V. Risk factors for acquisition of urinary tract infections caused by ciprofloxacin resistant Escherichia coli. J Urol 1995; 153: 117-20.

31.- Kahlmeter G, Menday P, Cars O. Nonhospital antimicrobial usage and resistance in community acquired Escherichia coli urinary tract infection. J Antimicrob Chemother 2003; 52: $1005-10$

32.- Wirtz V J, Dreser A, González R. Trends in antibiotic utilization in eight Latin American countries, 1997-2007. Rev Panam Salud Pública 2010; 27: 219-25.

33.- Ferech M, Coenen S, Malhotra-Kumar S, Dvorakova K, Hendrickx E, Suetens C, et al. European Surveillance of Antimicrobial Consumption (ESAC) outpatient quinolone use in Europe. J Antimicrob Chemother 2006; 58: 423-7.

34.- Ena J, López-Perezagua M M, MartínezPeinado C, Cia-Barrio MA, Ruíz-López I. Emergence of ciprofloxacin resistance in Escherichia coli isolates after widespread use of fluoroquinolones. Diagn Microbiol Infect Dis 1998; 30: 103-7.

35.- Smith S P, Manges A R, Riley L W. Temporal changes in the prevalence of communityacquired antimicrobial-resistant urinary tract infection affected by Escherichia coli clonal group composition. Clin Infect Dis 2008; 46: 689-95.

36.- Cagnacci S, Gualco L, Debbia E, Schito G C, Marchese A. European emergence of ciprofloxacin-resistant Escherichia coli clonal groups O25:H4-ST 131 and $\mathrm{O} 15: \mathrm{K} 52: \mathrm{H} 1$ causing community-acquired uncomplicated cystitis. J Clin Microbiol 2008; 46: 2605-12.

37.- Johnson J R, Menard M, Johnston B, Kuskowski M A, Nichol K, Zhanel G G Epidemic clonal groups of Escherichia coli as a cause of antimicrobial-resistant urinary tract infections in Canada, 2002 to 2004. Antimicrob Agents Chemother. 2009; 53: 2733-9.

38.- Vincent C, Boerlin P, Daignault D, Dozois C M, Dutil L, Galanakis C, et al. Food reservoir for Escherichia coli causing urinary tract infections. Emerg Infect Dis. 2010; 16: 88-95. 